\title{
The UTfit collaboration average of $D$ meson mixing data: Winter 2014
}

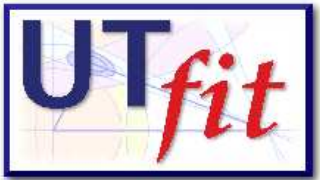

\section{The UTfit collaboration}

A.J. Bevan, ${ }^{a}$ M. Bona, ${ }^{a}$ M. Ciuchini, ${ }^{b}$ D. Derkach, ${ }^{c}$ E. Franco, ${ }^{d}$ V. Lubicz, ${ }^{b, e}$

G. Martinelli, ${ }^{d, f}$ F. Parodi, ${ }^{g}$ M. Pierini, ${ }^{h}$ C. Schiavi, ${ }^{g}$ L. Silvestrini, ${ }^{d}$ V. Sordini, ${ }^{i}$

A. Stocchi, ${ }^{j}$ C. Tarantino ${ }^{b, e}$ and V. Vagnoni ${ }^{k}$

${ }^{a}$ Queen Mary, University of London,

Mile End Road, London E1 4NS, U.K.

${ }^{b}$ INFN - Sezione di Roma Tre,

Via della Vasca Navale 84, I-00146 Roma, Italy

${ }^{c}$ Department of Physics, University of Oxford,

OX1 3PN Oxford, U.K.

${ }^{d}$ INFN - Sezione di Roma,

Piazzale A. Moro 2, I-00185 Roma, Italy

e Dipartimento di Matematica e Fisica, Università di Roma Tre,

Via della Vasca Navale 84, I-00146 Roma, Italy

${ }^{f} S I S S A-I S A S$,

Via Bonomea 265, I-34136 Trieste, Italy

${ }^{g}$ Dipartimento di Fisica, Università di Genova and INFN,

Via Dodecaneso 33, I-16146 Genova, Italy

${ }^{h} C E R N$,

CH-1211 Geneva 23, Switzerland

${ }^{i} I P N L-I N 2 P 3$,

4 Rue Enrico Fermi, F-69622 Villeurbanne Cedex, France

${ }^{j}$ Laboratoire de l'Accélérateur Linéaire, IN2P3-CNRS et Université de Paris-Sud, BP 34, F-91898 Orsay Cedex, France

${ }^{k}$ INFN - Sezione di Bologna,

Via Irnerio 46, I-40126 Bologna, Italy

E-mail: utfit-mail@cern.ch

Collaboration web page: http://www.utfit.org 
ABSTRACT: We update the analysis of $D$ meson mixing including the latest experimental results as of January 2014. We derive constraints on the parameters $M_{12}, \Gamma_{12}$ and $\Phi_{12}$ that describe $D$ meson mixing using all available data, allowing for $\mathrm{CP}$ violation. We also provide posterior distributions for observable parameters appearing in $D$ physics.

KeYwords: Beyond Standard Model, Heavy Quark Physics, CP violation

ARXIV EPRINT: 1402.1664 
Almost two years ago, we presented our combination of the $D$ mixing experimental data, yielding a quite precise determination of the mixing parameters showing no sign of CP violation [1]. Recently, the LHCb Collaboration has improved several important measurements $[2,3]$, and updates have also come from the other experiments [4-7]. These improvements result in a remarkable accuracy in the determination of the $\mathrm{CP}$ violating phase in charm mixing, implying strong contraints on possible extensions of the Standard Model (SM). An update of our fit is timely and can be of use for phenomenological analyses of physics beyond the SM.

In this letter, we perform a fit to the experimental data in table 1 following the statistical method described in ref. [24] improved with a Markov-chain Monte Carlo as implemented in the BAT library [25]. The following parameters are varied with flat priors in a sufficiently large range:

$$
x=\frac{\Delta m}{\Gamma}, \quad y=\frac{\Delta \Gamma}{2 \Gamma}, \quad\left|\frac{q}{p}\right|, \quad \delta_{K \pi}, \quad \delta_{K \pi \pi}, \quad R_{D},
$$

where $q$ and $p$ are defined as $\left|D_{L, S}\right\rangle=p\left|D^{0}\right\rangle \pm q\left|\bar{D}^{0}\right\rangle$ with $|p|^{2}+|q|^{2}=1, \delta_{K \pi(\pi)}$ is the strong phase difference between the amplitudes $A\left(\bar{D} \rightarrow K^{+} \pi^{-}\left(\pi^{0}\right)\right)$ and $A\left(D \rightarrow K^{+} \pi^{-}\left(\pi^{0}\right)\right)$ and

$$
R_{D}=\frac{\Gamma\left(D^{0} \rightarrow K^{+} \pi^{-}\right)+\Gamma\left(\bar{D}^{0} \rightarrow K^{-} \pi^{+}\right)}{\Gamma\left(D^{0} \rightarrow K^{-} \pi^{+}\right)+\Gamma\left(\bar{D}^{0} \rightarrow K^{+} \pi^{-}\right)} .
$$

We make the following assumptions in order to combine the measurements in table 1: i) we assume that Cabibbo allowed (CA) and doubly Cabibbo suppressed (DCS) decays are purely tree-level SM processes, neglecting direct $\mathrm{CP}$ violation; ii) we neglect the weak phase difference between these channels, which is of $\mathcal{O}\left(10^{-3}\right)$. One can then write the following equations $[1,26-30]$ :

$$
\begin{aligned}
\delta & =\frac{1-|q / p|^{2}}{1+|q / p|^{2}}, \quad \arg \left(\Gamma_{12} q / p\right)=\arg (y+i \delta x), \\
A_{M} & =\frac{|q / p|^{4}-1}{|q / p|^{4}+1}, \quad R_{M}=\frac{x^{2}+y^{2}}{2}, \\
\left(\begin{array}{c}
x_{f}^{\prime} \\
y_{f}^{\prime}
\end{array}\right) & =\left(\begin{array}{cc}
\cos \delta_{f} & \sin \delta_{f} \\
-\sin \delta_{f} \cos \delta_{f}
\end{array}\right)\left(\begin{array}{c}
x \\
y
\end{array}\right), \\
\left(x_{ \pm}^{\prime}\right)_{f} & =\left|\frac{q}{p}\right|^{ \pm 1}\left(x_{f}^{\prime} \cos \phi \pm y_{f}^{\prime} \sin \phi\right), \\
\left(y_{ \pm}^{\prime}\right)_{f} & =\left|\frac{q}{p}\right|^{ \pm 1}\left(y_{f}^{\prime} \cos \phi \mp x_{f}^{\prime} \sin \phi\right), \\
y_{\mathrm{CP}} & =\left(\left|\frac{q}{p}\right|+\left|\frac{p}{q}\right|\right) \frac{y}{2} \cos \phi-\left(\left|\frac{q}{p}\right|-\left|\frac{p}{q}\right|\right) \frac{x}{2} \sin \phi \\
A_{\Gamma} & =\left(\left|\frac{q}{p}\right|-\left|\frac{p}{q}\right|\right) \frac{y}{2} \cos \phi-\left(\left|\frac{q}{p}\right|+\left|\frac{p}{q}\right|\right) \frac{x}{2} \sin \phi, \\
\left(y_{\mathrm{CPA}}^{\prime}\right)_{f} & =\frac{\left(y_{+}^{\prime}\right)_{f}+\left(y_{-}^{\prime}\right)_{f}}{2} \\
\left(x_{\mathrm{CPA}}^{\prime}\right)_{f}^{2}+\left(y_{\mathrm{CPA}}^{\prime}\right)_{f}^{2} & =\frac{\left(x_{+}^{\prime}\right)_{f}^{2}+\left(x_{-}^{\prime}\right)_{f}^{2}+\left(y_{+}^{\prime}\right)_{f}^{2}+\left(y_{-}^{\prime}\right)_{f}^{2}}{2}
\end{aligned}
$$

valid for Cabibbo allowed and doubly Cabibbo suppressed final states. 


\begin{tabular}{|c|c|c|c|c|c|c|c|}
\hline Observable & Value & \multicolumn{5}{|c|}{ Correlation Coeff. } & Reference \\
\hline$y_{C P}$ & $(0.866 \pm 0.155) \%$ & & & & & & {$[4,5,8-11]$} \\
\hline$A_{\Gamma}$ & $(-0.014 \pm 0.052) \%$ & & & & & & {$[2,4,5,12]$} \\
\hline$x$ & $(0.79 \pm 0.29 \pm 0.08 \pm 0.12) \%$ & 1 & -0.007 & $-0.255 \alpha$ & 0.216 & & {$[13]$} \\
\hline$y$ & $(0.30 \pm 0.24 \pm 0.1 \pm 0.07) \%$ & -0.007 & 1 & $-0.019 \alpha$ & -0.280 & & {$[13]$} \\
\hline$|q / p|$ & $(0.96 \pm 0.21)$ & $-0.255 \alpha$ & $-0.019 \alpha$ & 1 & $-0.128 \alpha$ & & {$[13]$} \\
\hline$\phi$ & $(-2.5 \pm 10.5)^{\circ}$ & 0.216 & -0.280 & $-0.128 \alpha$ & 1 & & {$[13]$} \\
\hline$x$ & $(0.16 \pm 0.23 \pm 0.12 \pm 0.08) \%$ & 1 & 0.0615 & & & & {$[14]$} \\
\hline$y$ & $(0.57 \pm 0.20 \pm 0.13 \pm 0.07) \%$ & 0.0615 & 1 & & & & {$[14]$} \\
\hline$R_{M}$ & $(0.0130 \pm 0.0269) \%$ & & & & & & {$[15-19]$} \\
\hline$\left(x_{+}^{\prime}\right)_{K \pi \pi}$ & $(2.48 \pm 0.59 \pm 0.39) \%$ & 1 & -0.69 & & & & {$[20]$} \\
\hline$\left(y_{+}^{\prime}\right)_{K \pi \pi}$ & $(-0.07 \pm 0.65 \pm 0.50) \%$ & -0.69 & 1 & & & & {$[20]$} \\
\hline$\left(x_{-}^{\prime}\right)_{K \pi \pi}$ & $(3.50 \pm 0.78 \pm 0.65) \%$ & 1 & -0.66 & & & & {$[20]$} \\
\hline$\left(y_{-}^{\prime}\right)_{K \pi \pi}$ & $(-0.82 \pm 0.68 \pm 0.41) \%$ & -0.66 & 1 & & & & {$[20]$} \\
\hline$R_{D}$ & $(0.533 \pm 0.107 \pm 0.045) \%$ & 1 & 0 & 0 & -0.42 & 0.01 & {$[6]$} \\
\hline$x^{2}$ & $(0.06 \pm 0.23 \pm 0.11) \%$ & 0 & 1 & -0.73 & 0.39 & 0.02 & {$[6]$} \\
\hline$y$ & $(4.2 \pm 2 \pm 1) \%$ & 0. & -0.73 & 1 & -0.53 & -0.03 & {$[6]$} \\
\hline $\cos \delta_{K \pi}$ & $(0.84 \pm 0.2 \pm 0.06)$ & -0.42 & 0.39 & -0.53 & 1 & 0.04 & {$[6]$} \\
\hline $\sin \delta_{K \pi}$ & $(-0.01 \pm 0.41 \pm 0.04)$ & 0.01 & 0.02 & -0.03 & 0.04 & 1 & {$[6]$} \\
\hline$R_{D}$ & $(0.3030 \pm 0.0189) \%$ & 1 & 0.77 & -0.87 & & & {$[21]$} \\
\hline$\left(x_{+}^{\prime}\right)_{K \pi}^{2}$ & $(-0.024 \pm 0.052) \%$ & 0.77 & 1 & -0.94 & & & {$[21]$} \\
\hline$\left(y_{+}^{\prime}\right)_{K \pi}$ & $(0.98 \pm 0.78) \%$ & -0.87 & -0.94 & 1 & & & {$[21]$} \\
\hline$A_{D}$ & $(-2.1 \pm 5.4) \%$ & 1 & 0.77 & -0.87 & & & {$[21]$} \\
\hline$\left(x_{-}^{\prime}\right)_{K \pi}^{2}$ & $(-0.020 \pm 0.050) \%$ & 0.77 & 1 & -0.94 & & & {$[21]$} \\
\hline$\left(y_{-}^{\prime}\right)_{K \pi}$ & $(0.96 \pm 0.75) \%$ & -0.87 & -0.94 & 1 & & & {$[21]$} \\
\hline$R_{D}$ & $(0.364 \pm 0.018) \%$ & 1 & 0.655 & -0.834 & & & $\begin{array}{l}{[22]} \\
\end{array}$ \\
\hline$\left(x_{+}^{\prime}\right)_{K \pi}^{2}$ & $(0.032 \pm 0.037) \%$ & 0.655 & 1 & -0.909 & & & {$[22]$} \\
\hline$\left(y_{+}^{\prime}\right)_{K \pi}$ & $(-0.12 \pm 0.58) \%$ & -0.834 & -0.909 & 1 & & & {$[22]$} \\
\hline$A_{D}$ & $(2.3 \pm 4.7) \%$ & 1 & 0.655 & -0.834 & & & {$[22]$} \\
\hline$\left(x_{-}^{\prime}\right)_{K \pi}^{2}$ & $(0.006 \pm 0.034) \%$ & 0.655 & 1 & -0.909 & & & {$[22]$} \\
\hline$\left(y_{-}^{\prime}\right)_{K \pi}$ & $(0.20 \pm 0.54) \%$ & -0.834 & -0.909 & 1 & & & {$[22]$} \\
\hline$R_{D}$ & $(0.351 \pm 0.035) \%$ & 1 & -0.967 & 0.900 & & & {$[7]$} \\
\hline$\left(y_{\mathrm{CPA}}^{\prime}\right)_{K \pi}$ & $(0.43 \pm 0.43) \%$ & -0.967 & 1 & -0.975 & & & {$[7]$} \\
\hline$\left(x_{\mathrm{CPA}}^{\prime}\right)_{K \pi}^{2}$ & $(0.008 \pm 0.018) \%$ & 0.900 & -0.975 & 1 & & & {$[7]$} \\
\hline$R_{D}$ & $(0.3568 \pm 0.0058 \pm 0.0033) \%$ & 1 & -0.894 & 0.77 & -0.895 & 0.772 & {$[3]$} \\
\hline$\left(y_{+}^{\prime}\right)_{K \pi}$ & $(0.48 \pm 0.09 \pm 0.06) \%$ & -0.894 & 1 & -0.949 & 0.765 & -0.662 & {$[3]$} \\
\hline$\left(x_{+}^{\prime}\right)_{K \pi}^{2}$ & $(6.4 \pm 4.7 \pm 3) 10^{-5}$ & 0.77 & -0.949 & 1 & -0.662 & 0.574 & {$[3]$} \\
\hline$\left(y_{-}^{\prime}\right)_{K \pi}$ & $(0.48 \pm 0.09 \pm 0.06) \%$ & -0.895 & 0.765 & -0.662 & 1 & -0.95 & {$[3]$} \\
\hline$\left(x_{-}^{\prime}\right)_{K \pi}^{2}$ & $(4.6 \pm 4.6 \pm 3) 10^{-5}$ & 0.772 & -0.662 & 0.574 & -0.95 & 1 & {$[3]$} \\
\hline
\end{tabular}

Table 1. Experimental data used in the analysis, from ref. [23] and online updates at http://www.slac.stanford.edu/xorg/hfag/. $\alpha=(1+|q / p|)^{2} / 2$. Asymmetric errors have been symmetrized. 


\begin{tabular}{|ccc|}
\hline parameter & result @ 68\% prob. & $95 \%$ prob. range \\
\hline$\left|M_{12}\right|\left[\mathrm{ps}^{-1}\right]$ & $(4.4 \pm 2.0) \cdot 10^{-3}$ & {$[0.3,7.7] \cdot 10^{-3}$} \\
$\left|\Gamma_{12}\right|\left[\mathrm{ps}^{-1}\right]$ & $(14.9 \pm 1.6) \cdot 10^{-3}$ & {$[11.7,18.5] \cdot 10^{-3}$} \\
$\Phi_{M_{12}}\left[^{\circ}\right]$ & $(2.0 \pm 2.7)$ & {$[-4,12]$} \\
\hline$\delta_{K \pi}\left[^{\circ}\right]$ & $(8 \pm 13)$ & {$[-22,30]$} \\
$\delta_{K \pi \pi}\left[^{\circ}\right]$ & $(-6 \pm 23)$ & {$[-50,43]$} \\
\hline$x$ & $(3.6 \pm 1.6) \cdot 10^{-3}$ & {$[0.3,6.7] \cdot 10^{-3}$} \\
$y$ & $(6.1 \pm 0.7) \cdot 10^{-3}$ & {$[4.8,7.6] \cdot 10^{-3}$} \\
$|q / p|$ & $1.016 \pm 0.018$ & {$[0.981,1.058]$} \\
$\delta$ & $(-1.6 \pm 1.8) \cdot 10^{-2}$ & {$[-5.7,1.9] \cdot 10^{-2}$} \\
$\phi\left[^{\circ}\right]$ & $-0.5 \pm 0.6$ & {$[-1.8,0.6]$} \\
$R_{D}$ & $(3.50 \pm 0.04) \cdot 10^{-3}$ & {$[3.43,3.57] \cdot 10^{-3}$} \\
$A_{\Gamma}$ & $(1.4 \pm 1.5) \cdot 10^{-4}$ & {$[-1.5,4.4] \cdot 10^{-4}$} \\
$R_{M}$ & $(2.4 \pm 0.6) \cdot 10^{-5}$ & {$[1.6,4.1] \cdot 10^{-5}$} \\
$A_{M}$ & $(3.2 \pm 3.6) \cdot 10^{-2}$ & {$[-3.8,11.3] \cdot 10^{-2}$} \\
$y_{\mathrm{CP}}$ & $(6.1 \pm 0.7) \cdot 10^{-3}$ & {$[4.8,7.6] \cdot 10^{-3}$} \\
\hline
\end{tabular}

Table 2. Results of the fit to $D$ mixing data.

In the standard CKM phase convention (taking $\mathrm{CP}|D\rangle=|\bar{D}\rangle$ ), within the approximation we are using, CA and DCS decay amplitudes have vanishing weak phase and $\phi=\arg (q / p)$. Given the present experimental accuracy, one can assume $\Gamma_{12}$ to be real, ${ }^{1}$ leading to the relation

$$
\phi=\arg (y+i \delta x) .
$$

For the purpose of constraining NP, it is useful to express the fit results in terms of the $\Delta C=2$ effective Hamiltonian matrix elements $M_{12}$ and $\Gamma_{12}$ :

$$
\begin{aligned}
\left|M_{12}\right| & =\frac{1}{\tau_{D}} \sqrt{\frac{x^{2}+\delta^{2} y^{2}}{4\left(1-\delta^{2}\right)}} \sim \frac{x}{2 \tau_{D}}+\mathcal{O}\left(\delta^{2}\right), \quad\left|\Gamma_{12}\right|=\frac{1}{\tau_{D}} \sqrt{\frac{y^{2}+\delta^{2} x^{2}}{1-\delta^{2}}} \sim \frac{y}{\tau_{D}}+\mathcal{O}\left(\delta^{2}\right), \\
\sin \Phi_{12} & =\frac{\left|\Gamma_{12}\right|^{2}+4\left|M_{12}\right|^{2}-\left(x^{2}+y^{2}\right)|q / p|^{2} / \tau_{D}^{2}}{4\left|M_{12} \Gamma_{12}\right|} \sim \frac{x^{2}+y^{2}}{x y} \delta+\mathcal{O}\left(\delta^{2}\right)
\end{aligned}
$$

with $\Phi_{12}=\arg \left(\Gamma_{12} / M_{12}\right)$ and $\tau_{D}=0.41$ ps. Consistently with the assumptions above, $\Gamma_{12}$ can be taken real with negligible NP contributions, and a nonvanishing $\Phi_{12}=-\Phi_{M_{12}}$ can be interpreted as a signal of new sources of CP violation in $M_{12}$.

The results of the fit are reported in table 2. The corresponding probability density functions (p.d.f.'s) are shown in figures 1 and 2. Some two-dimensional p.d.f.'s are displayed in figure 3.

\footnotetext{
${ }^{1}$ See ref. [31] for a discussion of the size of $\arg \left(\Gamma_{12}\right)$.
} 

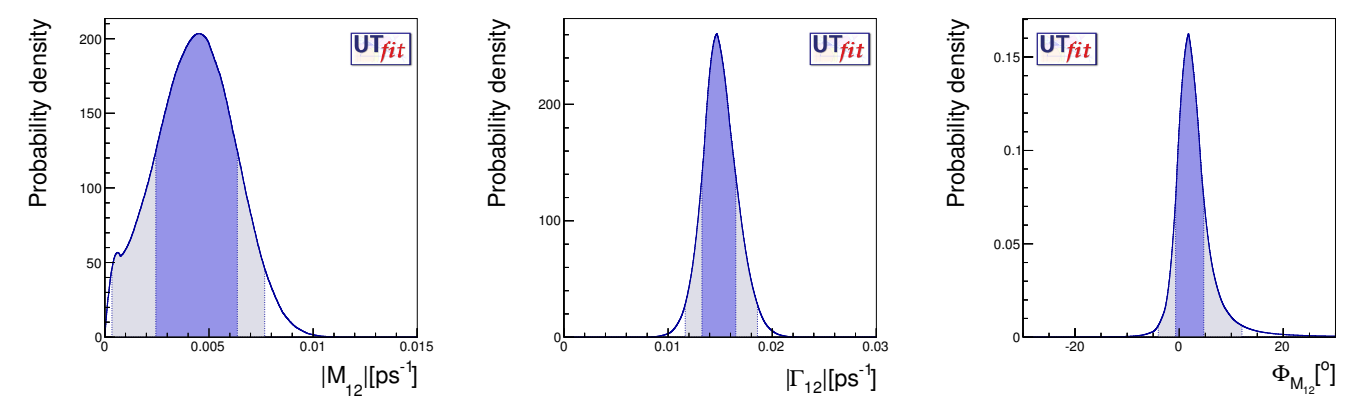

Figure 1. One-dimensional p.d.f. for the parameters $\left|M_{12}\right|,\left|\Gamma_{12}\right|$ and $\Phi_{M_{12}}$.
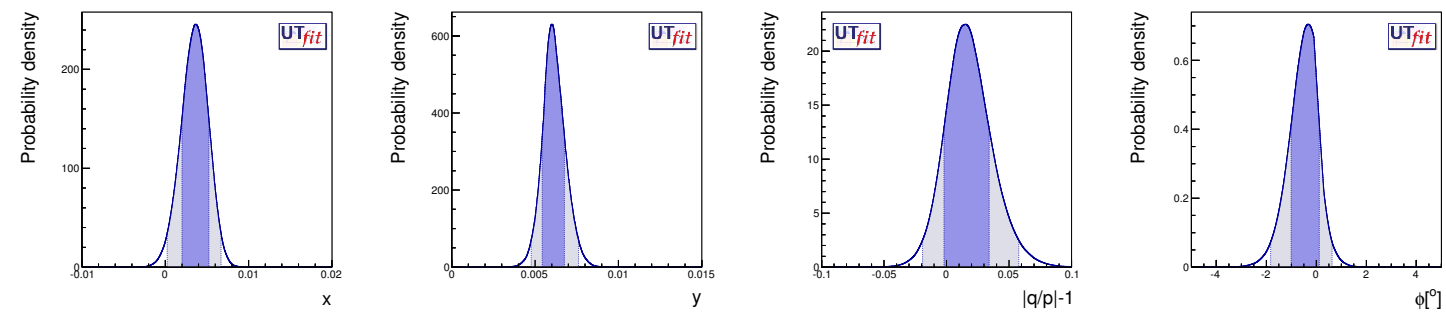

Figure 2. One-dimensional p.d.f. for the parameters $x, y,|q / p|-1$ and $\phi$.
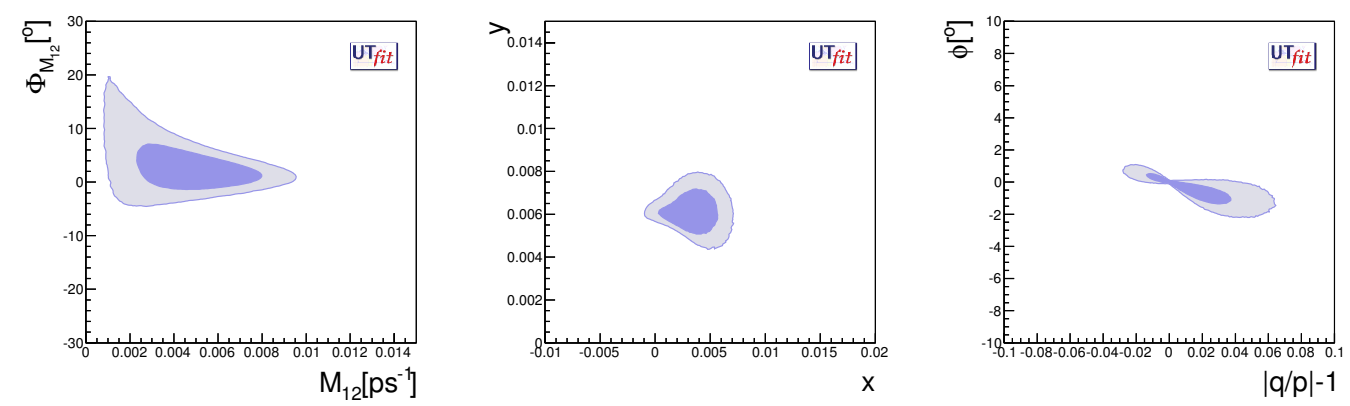

Figure 3. Two-dimensional p.d.f. for $\Phi_{12}$ vs $\left|M_{12}\right|$ (left), $y$ vs $x$ (middle) and $\phi$ vs $|q / p|-1$ (right).

As can be seen from table 2, the fitted value of $\delta$ is at the percent level and indeed the central values of $\left|M_{12}\right|,\left|\Gamma_{12}\right|$ and $\Phi_{12}$ are compatible with the expanded formulae in eq. (4). However in our fit we used the exact formulae since the region of $x \lesssim 10^{-4}$, still allowed by experimental data (although with probability less than $5 \%$ ), breaks the validity of the small $\delta$ expansion.

The results in table 2 can be used to constrain NP contributions to $D-\bar{D}$ mixing and decays.

Our results are in very good agreement with the fit labeled "No direct CPV in DCS decays" by HFAG [23] and online updates at http://www.slac.stanford.edu/xorg/hfag/, now that HFAG uses the theoretical relation in eq. (3) as we suggested in our previous paper. 


\section{Acknowledgments}

M.C. is associated to the Dipartimento di Matematica e Fisica, Università di Roma Tre. E.F. and L.S. are associated to the Dipartimento di Fisica, Università di Roma "La Sapienza". The research leading to these results has received funding from the European Research Council under the European Union's Seventh Framework Programme (FP/20072013)/ERC Grant Agreements n. 279972 "NPFlavour", n. 267985 "DaMeSyFla" and from the People Programme (Marie Curie Actions) under European Union's Seventh Framework Programme (FP7/2007-2013)/REA Grant Agreement n. 329017 "Charm@LHCb".

Open Access. This article is distributed under the terms of the Creative Commons Attribution License (CC-BY 4.0), which permits any use, distribution and reproduction in any medium, provided the original author(s) and source are credited.

\section{References}

[1] UTfit collaboration, A. Bevan et al., The UTfit collaboration average of D meson mixing data: Spring 2012, JHEP 10 (2012) 068 [arXiv: 1206.6245] [INSPIRE].

[2] LHCb collaboration, Measurements of indirect CP asymmetries in $D^{0} \rightarrow K^{-} K^{+}$and $D^{0} \rightarrow \pi^{-} \pi^{+}$decays, Phys. Rev. Lett. 112 (2014) 041801 [arXiv:1310.7201] [INSPIRE].

[3] LHCb collaboration, Measurement of $D^{0}-\bar{D}^{0}$ mixing parameters and search for CP-violation using $D^{0} \rightarrow K^{+} \pi^{-}$decays, Phys. Rev. Lett. 111 (2013) 251801 [arXiv:1309.6534] [INSPIRE].

[4] BABAR collaboration, J. Lees et al., Measurement of $D^{0}-\bar{D}^{0}$ mixing and CP-violation in two-body $D^{0}$ decays, Phys. Rev. D 87 (2013) 012004 [arXiv: 1209.3896] [InSPIRE].

[5] Belle collaboration, M. Staric, New Belle results on $D^{0}-\bar{D}^{0}$ mixing, arXiv:1212.3478 [INSPIRE].

[6] CLEO collaboration, D. Asner et al., Updated measurement of the strong phase in $D^{0} \rightarrow K^{+} \pi^{-}$decay using quantum correlations in $e^{+} e^{-} \rightarrow D^{0} \bar{D}^{0}$ at CLEO, Phys. Rev. D 86 (2012) 112001 [arXiv:1210.0939] [INSPIRE].

[7] CDF collaboration, T.A. Aaltonen et al., Observation of $D^{0}-\bar{D}^{0}$ mixing using the CDF II detector, Phys. Rev. Lett. 111 (2013) 231802 [arXiv:1309.4078] [INSPIRE].

[8] FOCUS collaboration, J. Link et al., A measurement of lifetime differences in the neutral $D$ meson system, Phys. Lett. B 485 (2000) 62 [hep-ex/0004034] [INSPIRE].

[9] CLEO collaboration, S. Csorna et al., Lifetime differences, direct CP-violation and partial widths in $D^{0}$ meson decays to $K^{+} K^{-}$and $\pi^{+} \pi^{-}$, Phys. Rev. D 65 (2002) 092001 [hep-ex/0111024] [INSPIRE].

[10] BeLLe collaboration, A. Zupanc et al., Measurement of $y_{C P}$ in $D^{0}$ meson decays to the $K_{S}^{0} K^{+} K^{-}$final state, Phys. Rev. D 80 (2009) 052006 [arXiv:0905.4185] [INSPIRE].

[11] LHCb collaboration, Measurement of mixing and CP-violation parameters in two-body charm decays, JHEP 04 (2012) 129 [arXiv:1112.4698] [InSPIRE].

[12] E791 collaboration, E. Aitala et al., Measurements of lifetimes and a limit on the lifetime difference in the neutral D meson system, Phys. Rev. Lett. 83 (1999) 32 [hep-ex/9903012] [INSPIRE]. 
[13] BELLE collaboration, K. Abe et al., Measurement of $D^{0}-\bar{D}^{0}$ mixing parameters in $D^{0} \rightarrow K_{S}^{0} \pi^{+} \pi^{-}$decays, Phys. Rev. Lett. 99 (2007) 131803 [arXiv:0704.1000] [InSPIRE].

[14] BABAR collaboration, P. del Amo Sanchez et al., Measurement of $D^{0}-\bar{D}^{0}$ mixing parameters using $D^{0} \rightarrow K_{S}^{0} \pi^{+} \pi^{-}$and $D^{0} \rightarrow K_{S}^{0} K^{+} K^{-}$decays, Phys. Rev. Lett. 105 (2010) 081803 [arXiv: 1004.5053] [INSPIRE].

[15] E791 collaboration, E. Aitala et al., Search for $D^{0}-\bar{D}^{0}$ mixing in semileptonic decay modes, Phys. Rev. Lett. 77 (1996) 2384 [hep-ex/9606016] [INSPIRE].

[16] CLEO collaboration, C. Cawlfield et al., Limits on neutral D mixing in semileptonic decays, Phys. Rev. D 71 (2005) 077101 [hep-ex/0502012] [INSPIRE].

[17] BABAR collaboration, B. Aubert et al., Search for $D^{0}-\bar{D}^{0}$ mixing using semileptonic decay modes, Phys. Rev. D 70 (2004) 091102 [hep-ex/0408066] [INSPIRE].

[18] BABAR collaboration, Search for $D^{0}-\bar{D}^{0}$ mixing using doubly flavor tagged semileptonic decay modes, Phys. Rev. D 76 (2007) 014018 [arXiv:0705.0704] [INSPIRE].

[19] BELLE collaboration, U. Bitenc et al., Improved search for $D^{0}-\bar{D}^{0}$ mixing using semileptonic decays at Belle, Phys. Rev. D 77 (2008) 112003 [arXiv:0802.2952] [INSPIRE].

[20] BABAR collaboration, B. Aubert et al., Measurement of $D^{0}-\bar{D}^{0}$ mixing from a time-dependent amplitude analysis of $D^{0} \rightarrow K^{+} \pi^{-} \pi^{0}$ decays, Phys. Rev. Lett. 103 (2009) 211801 [arXiv: 0807 .4544] [INSPIRE].

[21] BaBAr collaboration, B. Aubert et al., Evidence for $D^{0}-\bar{D}^{0}$ mixing, Phys. Rev. Lett. 98 (2007) 211802 [hep-ex/0703020] [INSPIRE].

[22] BELLE collaboration, L. Zhang et al., Improved constraints on $D^{0}-\bar{D}^{0}$ mixing in $D^{0} \rightarrow K^{+} \pi^{-}$decays at BELLE, Phys. Rev. Lett. 96 (2006) 151801 [hep-ex/0601029] [INSPIRE].

[23] Heavy Flavor Averaging Group collaboration, Y. Amhis et al., Averages of B-hadron, C-hadron and $\tau$-lepton properties as of early 2012, arXiv:1207.1158 [INSPIRE].

[24] M. Ciuchini et al., 2000 CKM triangle analysis: A Critical review with updated experimental inputs and theoretical parameters, JHEP 07 (2001) 013 [hep-ph/0012308] [INSPIRE].

[25] A. Caldwell, D. Kollar and K. Kroninger, BAT: the Bayesian Analysis Toolkit, Comput. Phys. Commun. 180 (2009) 2197 [arXiv:0808.2552] [INSPIRE].

[26] G.C. Branco, L. Lavoura and J.P. Silva, CP violation, Int. Ser. Monogr. Phys. 103 (1999) 1 [INSPIRE].

[27] G. Raz, Interpreting experimental bounds on $D^{0}-\bar{D}^{0}$ mixing in the presence of $C P$-violation, Phys. Rev. D 66 (2002) 057502 [hep-ph/0205113] [INSPIRE].

[28] M. Ciuchini et al., D- $\bar{D}$ mixing and new physics: general considerations and constraints on the MSSM, Phys. Lett. B 655 (2007) 162 [hep-ph/0703204] [INSPIRE].

[29] A.L. Kagan and M.D. Sokoloff, On indirect $C P$-violation and implications for $D^{0}-\bar{D}^{0}$ and $B_{s}-\bar{B}_{s}$ mixing, Phys. Rev. D 80 (2009) 076008 [arXiv:0907.3917] [InSPIRE].

[30] Y. Grossman, Y. Nir and G. Perez, Testing new indirect CP-violation, Phys. Rev. Lett. 103 (2009) 071602 [arXiv:0904.0305] [INSPIRE].

[31] A. Kagan, $C P$ violation in $D-\bar{D}$ mixing: absorptive contributions in the standard model, talk presented at the Workshop on $\tau$-Charm At High Luminosity, May 26-31, Isola d'Elba, Italy (2013). 